\title{
Timing-dependent effects of transcranial direct current stimulation with mirror therapy on daily function and motor control in chronic stroke: a randomized controlled pilot study
}

Wan-wen Liao ${ }^{1 \dagger}$, Wei-chi Chiang ${ }^{2 \dagger}$, Keh-chung Lin ${ }^{3,4}$, Ching-yi Wu ${ }^{1,5,6^{*}}$ (D), Chien-ting Liu', Yu-wei Hsieh ${ }^{1,5,6}$, Yun-chung Lin ${ }^{8}$ and Chia-ling Chen ${ }^{6,9}$

\begin{abstract}
Background: The timing of transcranial direct current stimulation (tDCS) with neurorehabilitation interventions may affect its modulatory effects. Motor function has been reported to be modulated by the timing of tDCS; however, whether the timing of tDCS would also affect restoration of daily function and upper extremity motor control with neurorehabilitation in stroke patients remains largely unexplored. Mirror therapy (MT) is a potentially effective neurorehabilitation approach for improving paretic arm function in stroke patients. This study aimed to determine whether the timing of tDCS with MT would influence treatment effects on daily function, motor function and motor control in individuals with chronic stroke.

Methods: This study was a double-blinded randomized controlled trial. Twenty-eight individuals with chronic stroke received one of the following three interventions: (1) sequentially combined tDCS with MT (SEQ), (2) concurrently combined tDCS with MT (CON), and (3) sham tDCS with MT (SHAM). Participants received interventions for $90 \mathrm{~min} /$ day, 5 days/week for 4 weeks. Daily function was assessed using the Nottingham Extended Activities of Daily Living Scale. Upper extremity motor function was assessed using the Fugl-Meyer Assessment Scale. Upper extremity motor control was evaluated using movement kinematic assessments.

Results: There were significant differences in daily function between the three groups. The SEQ group had greater improvement in daily function than the CON and SHAM groups. Kinematic analyses showed that movement time of the paretic hand significantly reduced in the SEQ group after interventions. All three groups had significant improvement in motor function from pre-intervention to post-intervention.
\end{abstract}

(Continued on next page)

\footnotetext{
* Correspondence: cywu@mail.cgu.edu.tw

${ }^{+}$Wan-wen Liao and Wei-chi Chiang contributed equally to this work.

'Department of Occupational Therapy and Graduate Institute of Behavioral Sciences, College of Medicine, Chang Gung University, 259 Wen-hwa 1st Road, Taoyuan City, Taiwan

${ }^{5}$ Healthy Aging Research Center, Chang Gung University, Taoyuan, Taiwan

Full list of author information is available at the end of the article
}

C C The Author(s). 2020 Open Access This article is licensed under a Creative Commons Attribution 4.0 International License, which permits use, sharing, adaptation, distribution and reproduction in any medium or format, as long as you give appropriate credit to the original author(s) and the source, provide a link to the Creative Commons licence, and indicate if changes were made. The images or other third party material in this article are included in the article's Creative Commons licence, unless indicated otherwise in a credit line to the material. If material is not included in the article's Creative Commons licence and your intended use is not permitted by statutory regulation or exceeds the permitted use, you will need to obtain permission directly from the copyright holder. To view a copy of this licence, visit http://creativecommons.org/licenses/by/4.0/ The Creative Commons Public Domain Dedication waiver (http://creativecommons.org/publicdomain/zero/1.0/) applies to the data made available in this article, unless otherwise stated in a credit line to the data. 
(Continued from previous page)

Conclusion: The timing of tDCS with MT may influence restoration of daily function and movement efficiency of the paretic hand in chronic stroke patients. Sequentially applying tDCS prior to MT seems to be advantageous for enhancing daily function and hand movement control, and may be considered as a potentially useful strategy in future clinical application.

Trial registration: ClinicalTrials.gov Identifier: NCT02827864. Registered on 29th June, 2016.

Keywords: Timing-dependent effect, Transcranial direct current stimulation, Mirror therapy, Stroke, Activities of daily living, Upper extremity kinematics

\section{Introduction}

Stroke remains one of the leading causes of long-term disability [1]. Most stroke patients have difficulties performing every day activities due to paresis of upper limbs, which results in impaired activities of daily living (ADL) and reduced quality of life [2, 3]. Identifying strategies that can facilitate functional recovery is thus an important goal for stroke rehabilitation. In recent years, several neurorehabilitation approaches have been developed to augment functional recovery, for example repetitive, task-oriented training and non-invasive brain stimulation (NIBS) $[4,5]$. Repetitive, task-oriented training emphasizes repetitive practice of task-related arm movements to facilitate motor relearning and restore correct movement patterns [6]. On the other hand, noninvasive brain simulation aims to maximize brain plasticity by externally applying electrical stimulation to modulate cortical excitability [7]. Since these two types of approaches individually have been shown to improve stroke recovery, it has been proposed that a synergistic approach that combines both of them may further augment overall treatment effects $[8,9]$.

Mirror therapy (MT) is one type of repetitive taskoriented training that has been widely used in clinical and research settings [10]. During MT training, a mirror is positioned in between the paretic and non-paretic arm. The paretic arm is behind the mirror and participants can only see the non-paretic arm and its mirror reflection. Participants are required to focus their attention on the mirror reflection and imagine it is the paretic arm while performing bilateral movements as simultaneously as possible. This mirrored visual feedback is hypothesized to restore the efferent-afferent loop that is damaged after stroke and facilitate re-learning of correct movement patterns [11]. MT has been demonstrated to reduce arm impairment and improve sensorimotor function and quality of life in individuals with stroke [10-13].

Transcranial direct current stimulation (tDCS) is a commonly used NIBS technique in stroke rehabilitation. tDCS applies weak direct current to the scalp to modulate brain excitability [14]. This weak direct current gradually changes neural membrane potentials to facilitate depolarization (excitation) or hyper-polarization (inhibition) of the neurons to enhance plasticity of the brain [15]. tDCS has been demonstrated to modulate neural networks and enhance motor learning in stroke patients [7, 16-18]. Although tDCS can be used alone, it is often combined with other rehabilitation approaches to boost responses of the brain to therapies $[8,19,20]$. A recent meta-analysis further showed that combining tDCS with rehabilitation interventions could produce greater treatment effects on recovery of motor function than tDCS alone in stroke patients [21].

Combining tDCS with MT is a potentially promising approach to not only augment neural responses of the brain but also increase treatment benefits of MT. Nevertheless, one crucial factor that needs to be considered when combining tDCS with MT is the timing of tDCS [22]. tDCS can be applied prior to MT (i.e., offline tDCS) or concurrently with MT (i.e., online tDCS). To our knowledge, only two studies have examined the synergistic effects of combined tDCS with MT in chronic stroke patients [23, 24]. Cho et al. (2015) applied tDCS prior to MT or motor training without mirror reflection. They found significant improvements in manual dexterity and grip strength in the combined tDCS with MT group, suggesting that sequentially applying tDCS prior to MT could improve motor function. By contrast, Jin et al. (2019) delivered tDCS prior to or concurrently with MT and found advantageous effects on hand function in the concurrent $\mathrm{tDCS}$ with MT group. The conflicting results between these two studies indicated further needs to explore the interaction effects of the timing of tDCS with MT to determine the optimal combination strategy.

The important factor to consider when examining the effects of combined tDCS with MT is the treatment outcomes, especially for outcomes that are related to daily activities. ADL such as the basic ADL and complex instrumental ADL (IADL) are essential for independent living and well-being of stroke patients. Therefore, restoring daily function should be one of the priority goals of stroke rehabilitation. However, the previous two studies only examined the effects of combined tDCS with MT on motor function [23, 24]. No studies to date have examined the timing-dependent effects of tDCS with MT on daily function in chronic stroke patients. 
Whether the timing of tDCS can affect restoration of daily function with MT remains uncertain.

In addition to daily function, investigating arm movement kinematics changes with respect to the timing of tDCS with MT is also critical for determining the optimal combination strategy. Movement kinematics of the arms can provide information of whether true behavioral changes or compensation strategies occur during training $[25,26]$. However, the two previous studies included only clinical motor function measurements $[23,24]$. While these clinical measurements can inform clinicians/researchers of motor function changes, they may not necessarily capture spatial and temporal characteristics of movement as well as motor control strategies changes after the combined interventions [26, 27]. Assessing movement kinematics changes with respect to the timing of tDCS with MT would help to unravel the benefits of combined approach on motor control of the paretic arm.

The purpose of this study was to examine the timingdependent effects of tDCS with MT on daily function, upper extremity motor function and motor control in chronic stroke patients. The tDCS was applied sequentially prior to MT (i.e., sequentially combined tDCS with MT group, SEQ) or concurrently with MT (i.e., concurrently combined tDCS with MT, CON). The sham tDCS with MT was used as the control condition. In addition to motor function outcomes, we further included the ADL/IADL measurement and movement kinematics assessments. We hypothesized that the SEQ and COM groups would demonstrate differential improvements in daily function, motor function and motor control.

\section{Methods}

\section{Participants}

Individuals with stroke were recruited from medical centers in Taiwan. The inclusion criteria were (1) a firstever unilateral stroke, (2) Age above 18 years old, (3) stroke onset more than 6 months, (4) Fugl-Meyer assessment (FMA) scores between 20 and 56, indicating moderate to mild impairments [28], (5) no severe muscle spasticity at the paretic arm at all joints (the Modified Ashworth Scale scores $<3$ ), and (6) adequate cognitive function to follow instructions (the Mini Mental State Examination 224 ). The exclusion criteria were (1) participation in any drug or rehabilitation projects/experiments in the past 6 months, (2) had Botulinum toxin injections in the past 3 months, (3) severe vision or visual perception impairments (e.g., neglect and poor visual field) as assessed by the National Institutes of Health Stroke Subscale, (4) concomitant neurologic, neuromuscular or orthopedic conditions such as brain tumor and Parkinson's disease, and (5) any contradictions to NIBS [29]. All participants gave their written informed consent before participating in this study. This study was approved by the Institutional Review Board of Chang Gung Memorial Hospital, Taoyuan, Taiwan. All study procedures were conducted in accordance with the Declaration of Helsinki.

\section{Design}

This study was a double-blinded, randomized controlled trial with pre-intervention and post-intervention assessments (Fig. 1). Participants were stratified based on their initial upper extremity impairment levels (FMA scores $20-35$ vs. 36-56) [30] and randomly allocated to 3 groups: (1) sequential combination of tDCS with MT (SEQ), (2) concurrent combination of tDCS with MT $(\mathrm{CON})$, and (3) sham tDCS with MT (SHAM). Randomization procedures were performed using a randomization table generated online (freely available at http://www.randomizer.org/).

Participants were assessed within 1 week before and after interventions by the same raters that were not involved in training and blinded to the purpose and group allocation of this study. These raters were trained by senior occupational therapists and the principal investigator of this study to ensure they performed the assessments in a uniformed and standardized way. Outcome measures included clinical and kinematic assessments. The clinical and kinematic assessments were performed on two separate days within 1 week before and after interventions to minimize feeling of fatigue during assessments.

\section{Intervention protocols}

All participants received one of the three interventions for $90 \mathrm{~min} /$ day, 5 days/week, for 4 weeks. The intervention flow was illustrated in Fig. 2. The SEQ group received $20 \mathrm{~min}$ of anodal tDCS over the ipsilesional primary motor cortex (iM1) followed by $20 \mathrm{~min}$ of MT with sham tDCS and $20 \mathrm{~min}$ of MT alone. The CON group received sham tDCS during the first $20 \mathrm{~min}$, followed by $20 \mathrm{~min}$ of MT concurrently with anodal tDCS on iM1 and 20 min of MT alone. The sham tDCS in the SEQ and CON groups was used to keep the tDCS setting consistent between SEQ and CON conditions to blind participants from group allocation and prevent them from noticing any differences in tDCS settings. For the SHAM group, the training/stimulation procedures were the same as those for the other two groups, except that there was only sham tDCS provided. There were 30 min of functional task practice performed after MT for all three groups.

\section{tDCS protocol}

A battery-driven direct current stimulator (neuroConn $\mathrm{GmbH}$, Ilmenau, Germany) was used for delivering 


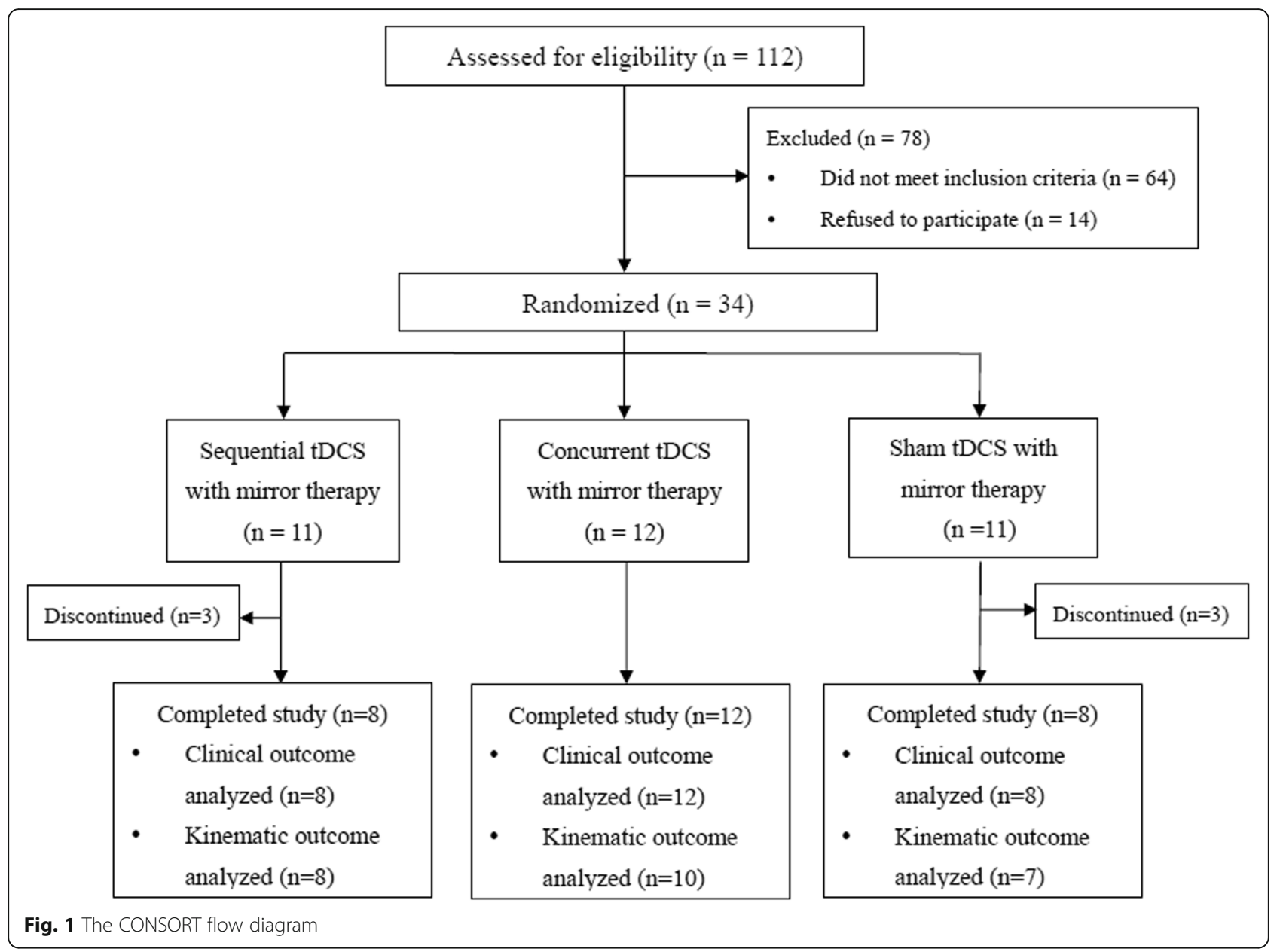

$20 \mathrm{~min} 20 \mathrm{~min} 20 \mathrm{~min}$

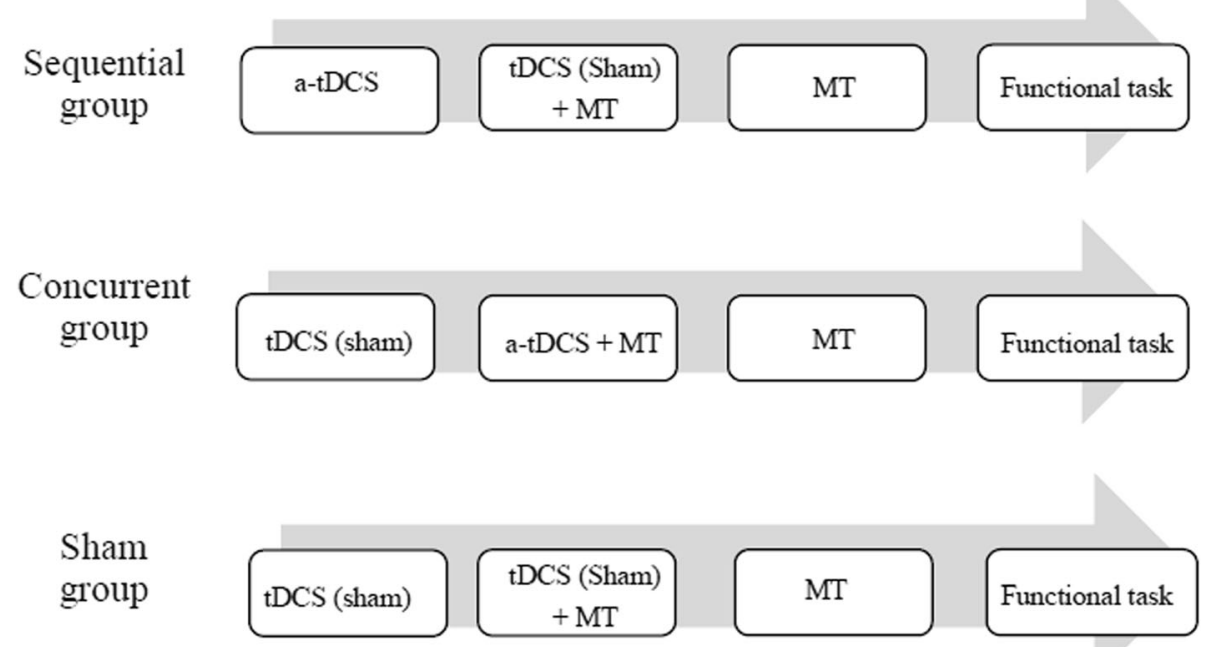

Fig. 2 Experimental flow of the three intervention groups. Note: a-tDCS, anodal tDCS; MT, mirror therapy; tDCS, transcranial direct current stimulation 
anodal tDCS. The anode electrode was placed over the $\mathrm{iM} 1$, which was the $\mathrm{C} 3 / \mathrm{C} 4$ location of the international 10-20 electroencephalogram (EEG) electrode system; while the cathode electrode was placed on the contralesional supraorbital area. The duration of tDCS was 20 $\min$. The size of the electrodes was $35 \mathrm{~cm}^{2}$, and the stimulation intensity was $2 \mathrm{~mA}$, resulting in a current density of $0.057 \mathrm{~mA} / \mathrm{cm}^{2}$ [31], which was well within the current safety limit [32]. For the sham tDCS, the stimulation intensity was first ramped up to $2 \mathrm{~mA}$ in $15 \mathrm{~s}$ and then ramped down to 0 within the next $30 \mathrm{~s}$ [33].

The tDCS protocols vary in the literature in terms of stimulation type, duration, intensity, and treatment sessions $[7,34]$. The anodal tDCS on the iM1 and the cathodal tDCS on contralesional M1 have been applied in stroke patients with a range of 10 to $30 \mathrm{~min}$ of stimulation and a range of stimulation intensity between 1 to 2 $\mathrm{mA}[7,34,35]$. The training sessions of combined tDCS with upper limb rehabilitation were between 14 to 30 sessions [7, 34, 35].

The tDCS protocol ( $2 \mathrm{~mA}$ of anodal tDCS for $20 \mathrm{~min}$ ) used in this study was developed based on evidence in the literature $[7,34,35]$. We decided to use anodal tDCS because studies have shown that the anodal tDCS produced a more consistent modulation effects than the cathodal tDCS [36, 37]. The inter-individual variability was also lower using the anodal tDCS than the cathodal tDCS $[36,37]$. Furthermore, anodal tDCS with an intensity of $2 \mathrm{~mA}$ and duration of $20 \mathrm{~min}$ was effective at increasing cortical excitability and producing long-lasting effects [37, 38]. Therefore, this tDCS protocol was implemented in the current study. Compared with the tDCS protocol of one previous study that examined the effects of combined tDCS with MT, the stimulation intensity and treatment sessions of our protocol were longer and only the lesioned cortex was stimulated in this study [23].

\section{MT training and functional task practice}

A mirror was placed in participants' sagittal plane during MT. Participants were required to look at the reflection of non-paretic arm in the mirror, imagined it as the paretic arm and performed bilateral arm movements as simultaneously as possible. The MT training consisted of (1) intransitive movements, including distal and proximal arm/hand movements such as wrist extension-flexion, forearm pronation-supination and elbow flexionextension, and (2) transitive movements, such as placing pegs in holes or flipping a card [13]. The functional task training focused on practice of daily activities that were meaningful and important for the participants. The contents of functional tasks were designed based on each participant's main complaints about the tasks that were difficult to perform during daily activities. Examples of functional task practice included stabilizing a bowl with the paretic hand and scooping food with the non-paretic hand, grasping a cup with the paretic hand and bringing the cup toward the mouth, or wringing out water from a wet towel with both hands. Common daily objects, such as cups, bowls or towels were used for functional task practice based on the requirements of the tasks. Participants practiced three different kinds of functional tasks in $30 \mathrm{~min} \mathrm{[13].}$

\section{Outcome measures \\ Clinical outcome assessments}

The Nottingham Extended Activities of Daily Living (NEADL) Scale was selected as the primary measure for evaluating the basic and instrumental ADL because it has been shown to have good psychometric properties (e.g. reliability and responsiveness) in stroke patients [39]. In addition, the NEADL does not have significant floor and celling effects in stroke patients and therefore it can be used in a wide range of stroke patients [40]. The NEADL consists of 22 items within four categories of daily activities, including mobility, kitchen, domestic, and leisure activities. The NEADL scale uses a 4-point rating scale. Higher scores indicate greater functional independence.

The Fugl-Meyer assessment scale of upper extremity (FMA) was used to assess sensorimotor function of upper limbs in individuals with stroke [41]. FMA consists of 33 movements with scores ranging from 0 to 66 . Higher FMA scores indicate less impairment of the paretic arm. The validity and reliability of FMA are good to excellent in stroke patients [42].

\section{Movement kinematic assessments}

A unilateral reaching task was used to assess movement kinematics of the paretic arm and trunk. Participants were seated in front of a table with the seat height adjusted to $100 \%$ of the lower leg length. Participants placed their paretic hands at a marked starting point at the edge of table with their elbow flexed at 90 degrees. During the unilateral reaching task, participants were required to reach to press a doorbell that was placed along their midsagittal plane at a distance of 1.25 times the arm length (defined as the distance between the medial border of the axilla and the midpoint of the styloid processes of ulna and radius) as quickly as they could.

A seven-camera motion analysis system (VICON MX; Oxford Metrics Inc., Oxford, England) was used to capture motions of the paretic arm and the trunk. Reflective markers were attached to the 7 th cervical vertebra (C7) and 4th thoracic vertebra (T4) spinal processes, mid sternum, bilateral clavicular head, acromion of the shoulder, anterior aspect of the upper arm midway between the acromion and lateral epicondyle, lateral 
epicondyle, ulnar and radial styloid processes, and the tip of thumb and index finger. Movements were recorded at $120 \mathrm{~Hz}$ and low-pass filtered at $5 \mathrm{~Hz}$ using a 2nd order Butterworth filter. A customized LabVIEW program (National Instruments Inc., Austin, TX) was used to process the kinematic data.

Kinematic outcome variables included the reaction time (RT), movement time (MT) and normalized total displacement (NTD). The hand marker (index finger) represented endpoint control and the sternal marker represented trunk control. Movement onset and offset were defined as the time point when the tangential velocity of the index finger/trunk rose above 5\% and fell below $5 \%$ of the peak velocity of that trial [43]. RT was defined as the interval from the start signal to movement onset [44] and represented temporal efficiency of generation and planning of actions. Movement time was defined as the duration between movement onset and offset and represented movement efficiency. Hand NTD was defined as the path length of the hand, and normalized by the straight distance between the hand at the start position and the target for each participant. Trunk NTD was defined as the displacement of the sternal marker from the initial position, and normalized by the straight distance from the initial to end position for each participant. NTD represented movement straightness [45]. A smaller value of NTD indicated a more straight movement path.

\section{Statistical analysis}

The chi-square test and the analysis of variance were used to examine baseline demographic and clinical characteristics of participants between groups. A paired t-test was used to compare differences from pre-intervention to post-intervention for each group. Analysis of covariance was used to evaluate differences of treatment effects between groups. The pre-intervention scores of each outcome were treated as the covariate to control for potential baseline differences. The effect size of partial eta squared $\left(\eta^{2}\right)$ was calculated for all outcome variables and represented the magnitude of changes between groups. $\eta^{2}$ greater than 0.138 represented a large effect. $\eta^{2}$ greater than 0.059 represented a moderate effect, and $\eta^{2}$ greater than 0.01 represented a small effect [46]. The effect size $d$ was also calculated for all outcomes and represented the magnitude of changes from pre- to post-intervention in each group [46]. Data were analyzed using SPSS 19.0 (IBM Corp., Armonk, NY). The alpha level was set at 0.05 .

\section{Results}

Thirty-four participants were enrolled in the study. Three participants in the SEQ group and three participants in the SHAM group did not complete interventions due to unexpected family events, lack of transportation, work responsibilities, childcare commitments and no-show. Therefore, twenty-eight participants completed all training sessions. There were eight participants in the SEQ group, twelve participants in the CON group and eight participants in the SHAM group (Fig. 1).

Participants' baseline demographics and clinical characteristics did not significantly differ between groups (Table 1). All twenty-eight participants completed clinical assessments (i.e., FMA and NEADL) at preintervention and post-intervention. Among them, three participants (two participants in the CON group and one participant in the SHAM group) could not perform the unilateral reaching task correctly for kinematic assessments. To minimize potential measurement errors, these three participants did not undergo kinematic assessments. The kinematic data included 25 participants (Fig. 1).

Table 2 summarizes the descriptive and inferential statistics for within-group and between-group comparison of clinical and kinematic outcomes. For within group comparison, significant improvements were found in the NEADL scores from pre-intervention to postintervention in the SEQ $(t=3.18, P=0.02, d=0.84)$ and CON ( $t=3.6, P=0.004, d=0.22)$ groups. However, no changes were found in the NEADL scores from preintervention to post-intervention in the SHAM group $(t=0.47, P=0.65, d=0.05)$. All three groups had significant increase in the FMA scores from pre-intervention to post-intervention (SEQ, $t=4.36, P=0.003, d=0.39$; CON, $t=3.15, P=0.01, d=0.54$; SHAM, $t=3.15, P=$ $0.02, d=0.38$ ). For the kinematic outcomes, significant improvement was demonstrated in the index movement time of the SEQ group from pre-intervention to postintervention $(t=-2.38, P=0.04, d=0.3)$. No differences were found in other kinematics variables from preintervention to post-interventions in the three groups ( $t=-2.18$ to $1.63, P=0.07$ to $0.98, d<0.001$ to $d=1.01$ ).

For between group comparison, significant differences were found in the NEADL scores $\left(F_{(2,25)}=4.69, P=0.02\right.$, $\left.\eta^{2}=0.28\right)$. Post hoc analyses showed that the SEQ group had significantly greater increase in the NEADL scores than the CON $(P=0.05)$ and the SHAM $(P=0.006)$ groups. However, no differences were found in the NEADL scores between the CON and SHAM groups $(P=0.24)$. There were no differences in the FMA scores $\left(F_{(2,25)}=0.27, P=0.77, \eta^{2}=0.02\right)$ and kinematic variables $\left(F_{(2,22)}=0.15\right.$ to $0.9, P=0.42$ to $0.9, \eta^{2}=0.01$ to 0.08 ) between groups. Fig. 3 illustrates the significant changes in the clinical (i.e., NEADL and FMA) and kinematic (i.e., index finger movement time) outcomes.

\section{Discussion}

To our knowledge, this study was the first to examine timing-dependent effects of tDCS with MT on daily 
Table 1 Demographic and clinical characteristics of the study participants

\begin{tabular}{|c|c|c|c|c|c|}
\hline Variables & SEQ $(N=8)$ & $\operatorname{CON}(N=12)$ & SHAM $(N=8)$ & Statistic $^{a}$ & $P$ \\
\hline Age (year) & $60.18 \pm 4.84$ & $52.04 \pm 8.68$ & $56.45 \pm 9.88$ & 2.43 & 0.11 \\
\hline Gender (Male/Female) & $5 / 3$ & $8 / 4$ & $8 / 0$ & 3.78 & 0.15 \\
\hline Side of lesion (Right/Left) & $2 / 6[45]$ & $5 / 7$ & $0 / 8$ & 4.44 & 0.11 \\
\hline Onset time (months) & $19.63 \pm 12.28$ & $21.92 \pm 11.83$ & $38.13 \pm 36.98$ & 1.75 & 0.2 \\
\hline Hemorrhagic/Ischemic Stroke & $2 / 6$ & $5 / 7$ & $1 / 7$ & 2.07 & 0.36 \\
\hline FMA & $37.50 \pm 14.95$ & $36.33 \pm 7.91$ & $30.75 \pm 9.16$ & 0.95 & 0.4 \\
\hline MMSE & $28.25 \pm 1.67$ & $28.83 \pm 2.12$ & $27.38 \pm 2.56$ & 1.11 & 0.35 \\
\hline MAS & $0.39 \pm 0.17$ & $0.50 \pm 0.28$ & $0.61 \pm 0.24$ & 1.64 & 0.22 \\
\hline
\end{tabular}

${ }^{2}$ Statistic associated with the chi-square test for categorical variables and with the analysis of variance for continuous variables. Note: SEQ Sequential group, CON Concurrent group, SHAM Sham group, FMA Fugl-Meyer assessment scale of upper extremity, MMSE Mini-Mental State Examination, MAS Modified Ashworth Scale. Value is presented as mean \pm standard deviation (SD)

function and movement control in participants with chronic stroke. We found greater improvements in overall ADL/IADL function in the SEQ group compared to the CON and SHAM groups, indicating that the timing of tDCS may affect restoration of basic and complex ADL abilities for independent living in the community. Furthermore, kinematic analyses revealed that the index movement time significantly reduced only in the SEQ group, but not the CON and SHAM groups. This result indicated that the SEQ group could move the paretic hand more efficiently during unilateral reaching tasks after interventions. By contrast, the CON and SHAM group did not show such improvements in motor control of paretic hands after interventions. All three groups had significant improvements in motor function.

Consistent with our hypothesis, we found timingdependent effects of tDCS with MT. In particular, this timing-dependent effect was exhibited in ADL/IADL function, but not clinical motor function. Applying tDCS prior to MT led to greater benefits on daily function than applying tDCS concurrently with MT. Compared with motor tasks, the ADL/IADL tasks often involve higher levels of motor-cognitive and perceptual processes and require the ability to transfer the learned skills to daily tasks [47-49]. In this study, during the SEQ condition, the anodal tDCS may serve as a priming stimulus to enhance activity of the lesioned cortex. This priming stimulus would create a favorable/excitable environment of the brain which is beneficial for activating higher-order motor-cognitive processes during the consecutive MT [50-52]. This activated brain state accompanied by repetitive practice of MT might facilitate the cognitive-motor relearning processes and enhance generalization of learned motor skills [7, 53]. By contrast, applying tDCS concurrently with MT might generate motor/cognitive interference during the MT practice, and consequently affect the cognitive-motor relearning processes and generalization of learned skills to daily activities [53-55]. Studies have also reported that delivering tDCS concurrently with motor/cognitive trainings interfered or abolished the modulatory effects of tDCS [55-58]. Our results were in line with these previous findings suggesting that the effects of tDCS with MT may not be simply additive, and that applying tDCS prior to MT might be a potentially beneficial approach for improving ADL/IADL function more than concurrent tDCS with MT in chronic stroke patients.

Contrary to our hypothesis, motor function improved to a similar extent between groups. These results indicated that there might be potentially no timingdependent effects of $\mathrm{tDCS}$ with MT, and combination of tDCS with MT either sequentially or concurrently did not yield to additional benefits on motor function than MT alone. Though surprising, these results were similar to the findings of no add-on effects of tDCS with motorimagery based modality (e.g., brain-computer interface, $\mathrm{BCI})$ or motor training on clinical motor function in several previous studies [59-61]. A recent systematic review of randomized controlled trials of tDCS also did not find additional benefits of tDCS on improving motor function (i.e., FMA scores), but rather benefits of tDCS on enhancing ADL capacity [62].

However, our finding of similar levels of motor function improvements between the SEQ and CON groups was contrary to the results of one previous study that examined the effects of combined tDCS with MT [23]. In that study, the concurrent-tDCS group showed greater improvements in motor function than that of priortDCS and sham groups. Nevertheless, our study protocol was different from those of the previous study in several ways. First, the stimulation paradigm was different. We stimulated the ipsilesional primary motor cortex while the previous study modulated both ipsilesional and contralesional primary motor cortex. Second, the stimulation intensity was higher (i.e., $2 \mathrm{~mA}$ ) in our study than the previous study (i.e., $1 \mathrm{~mA}$ ). Third, our participants received longer duration of interventions (i.e., 20 sessions) than that of previous study (i.e., 10 sessions). It is 
Table 2 Descriptive and inferential statistics of clinical and kinematic outcomes

\begin{tabular}{|c|c|c|c|c|c|c|c|c|c|c|c|c|c|c|c|c|c|c|}
\hline \multirow{4}{*}{$\begin{array}{l} \\
\text { Clinical } \\
\text { Variables }\end{array}$} & \multicolumn{3}{|c|}{ Pretreatment } & \multicolumn{3}{|c|}{ Posttreatment } & \multirow{2}{*}{\multicolumn{3}{|c|}{ Between-group }} & \multicolumn{9}{|c|}{ Within-group } \\
\hline & & & & & & & & & & \multicolumn{3}{|l|}{ SEQ } & \multicolumn{3}{|l|}{ CON } & \multicolumn{3}{|l|}{ SHAM } \\
\hline & SEQ & CON & SHAM & SEQ & CON & SHAM & $F$ & P & $\eta^{2}$ & $t$ & P & d & $t$ & P & d & t & $P$ & d \\
\hline & $\begin{array}{l}\text { Mean } \pm \\
\text { SD }\end{array}$ & Mean \pm SD & $\begin{array}{l}\text { Mean } \\
\pm S D\end{array}$ & $\begin{array}{l}\text { Mean } \pm \\
\text { SD }\end{array}$ & Mean \pm SD & Mean \pm SD & & & & & & & & & & & & \\
\hline NEADL & $\begin{array}{l}28.00 \pm \\
7.82\end{array}$ & $\begin{array}{l}33.42 \pm \\
15.63\end{array}$ & $\begin{array}{l}23.88 \pm \\
8.82\end{array}$ & $\begin{array}{l}35.75 \pm \\
10.48\end{array}$ & $37.00 \pm 16.46$ & $24.38 \pm 9.98$ & 4.69 & $0.02^{*}$ & 0.28 & 3.18 & $0.02^{*}$ & 0.84 & 3.60 & $0.004^{*}$ & 0.22 & 0.47 & 0.65 & 0.05 \\
\hline FMA & $\begin{array}{l}37.50 \pm \\
14.95\end{array}$ & $\begin{array}{l}36.33 \pm \\
7.91\end{array}$ & $\begin{array}{l}30.75 \pm \\
9.16\end{array}$ & $\begin{array}{l}43.25 \pm \\
14.34\end{array}$ & $41.67 \pm 11.41$ & $34.50 \pm 10.46$ & 0.27 & 0.77 & 0.02 & 4.36 & $0.003^{*}$ & 0.39 & 3.15 & $0.01^{*}$ & 0.54 & 3.15 & $0.02^{*}$ & 0.38 \\
\hline \multicolumn{19}{|c|}{ Kinematic Variables } \\
\hline $\begin{array}{l}\text { Trunk RT } \\
\text { (sec) }\end{array}$ & $0.29 \pm 0.12$ & $\begin{array}{l}0.36 \pm \\
0.14\end{array}$ & $\begin{array}{l}0.29 \pm \\
0.10\end{array}$ & $\begin{array}{l}0.31 \pm \\
0.78\end{array}$ & $0.27 \pm 0.59$ & $0.29 \pm 0.07$ & 0.53 & 0.6 & 0.05 & 0.56 & 0.59 & 0.04 & -1.58 & 0.15 & 0.21 & 0.09 & 0.93 & $<0.0$ \\
\hline $\begin{array}{l}\text { Index RT } \\
\text { (sec) }\end{array}$ & $0.47 \pm 0.17$ & $\begin{array}{l}0.53 \pm \\
0.12\end{array}$ & $\begin{array}{l}0.52 \pm \\
0.20\end{array}$ & $\begin{array}{l}0.47 \pm \\
0.16\end{array}$ & $0.44 \pm 0.18$ & $0.40 \pm 0.09$ & 0.30 & 0.74 & 0.03 & -0.03 & 0.98 & $<0.001$ & -1.25 & 0.24 & 0.59 & -1.28 & 0.25 & 0.77 \\
\hline $\begin{array}{l}\text { Trunk MT } \\
\text { (sec) }\end{array}$ & $2.40 \pm 0.83$ & $\begin{array}{l}2.17 \pm \\
0.69\end{array}$ & $\begin{array}{l}2.27 \pm \\
1.24\end{array}$ & $\begin{array}{l}1.63 \pm \\
0.36\end{array}$ & $2.09 \pm 1.14$ & $1.72 \pm 0.58$ & 0.74 & 0.49 & 0.07 & -2.18 & 0.07 & 1.2 & -0.23 & 0.83 & 0.08 & -1.01 & 0.35 & 0.57 \\
\hline $\begin{array}{l}\text { Index MT } \\
\text { (sec) }\end{array}$ & $2.30 \pm 0.80$ & $\begin{array}{l}1.92 \pm \\
0.92\end{array}$ & $\begin{array}{l}1.88 \pm \\
1.14\end{array}$ & $\begin{array}{l}1.50 \pm \\
0.37\end{array}$ & $2.00 \pm 1.19$ & $1.66 \pm 0.50$ & 0.9 & 0.42 & 0.08 & -2.38 & $0.04^{*}$ & 0.3 & 0.21 & 0.84 & 0.08 & -0.42 & 0.69 & 0.25 \\
\hline Trunk NTD & $1.25 \pm 0.35$ & $\begin{array}{l}2.35 \pm \\
3.33\end{array}$ & $\begin{array}{l}1.09 \pm \\
0.04\end{array}$ & $\begin{array}{l}1.08 \pm \\
0.48\end{array}$ & $1.12 \pm 0.89$ & $1.16 \pm 0.19$ & 0.66 & 0.53 & 0.06 & -1.29 & 0.24 & 0.4 & -1.16 & 0.28 & 0.5 & 0.82 & 0.45 & 0.51 \\
\hline Index NTD & $1.99 \pm 0.55$ & $\begin{array}{l}1.76 \pm \\
0.28\end{array}$ & $\begin{array}{l}1.46 \pm \\
0.19\end{array}$ & $\begin{array}{l}1.55 \pm \\
0.28\end{array}$ & $1.68 \pm 0.26$ & $1.84 \pm 0.51$ & 0.15 & 0.87 & 0.01 & -1.63 & 0.15 & 1.01 & -0.59 & 0.57 & 0.3 & 1.63 & 0.16 & 0.98 \\
\hline
\end{tabular}

SEQ Sequential group, CON Concurrent group, SHAM Sham group, FMA Fugl-Meyer Assessment Scale of Upper extremity, NEADL Nottingham Extended Activities of Daily Living Scale, $R T$ reaction time, $M T$ movement time, NTD normalized total displacement. ${ }^{*} \leqq 0.05$

possible that these differences in tDCS and intervention protocols resulting in the differential findings on motor function between our study and the previous study [54]. This result also indicated that tDCS parameters such as intensity and duration as well as modules of stimulation might affect the interaction effects of tDCS with MT on motor function, and these factors need to be carefully considered during clinical application. Future studies could examine the timing-dependent effects of tDCS with MT using different tDCS stimulation parameters and intervention protocols to optimize treatment benefits on motor function.

Another important question of this study was whether there would be timing-dependent effects of tDCS with MT on movement control of paretic upper extremity. In addition to clinical outcomes, the kinematic assessments were also employed to objectively track changes of spatial and temporal control of upper extremity. We found that movement efficiency of the paretic hand significantly improved in the SEQ group after interventions. This finding was consistent with the evidence reported by Giacobee et al. (2013) [63]. In their study, wrist movement kinematics improved only when tDCS was applied sequentially prior to robotic training, but not concurrently with training. As a result, it is possible that the order of tDCS with neurorehabilitation may influence restoration of distal arm movement control such as the wrist and hand. In particular, sequentially combined tDCS with MT may have potentials to improve distal hand control. Our study also indicated that kinematic assessments were sensitive to the timing-dependent effects of tDCS with MT on motor control of the paretic

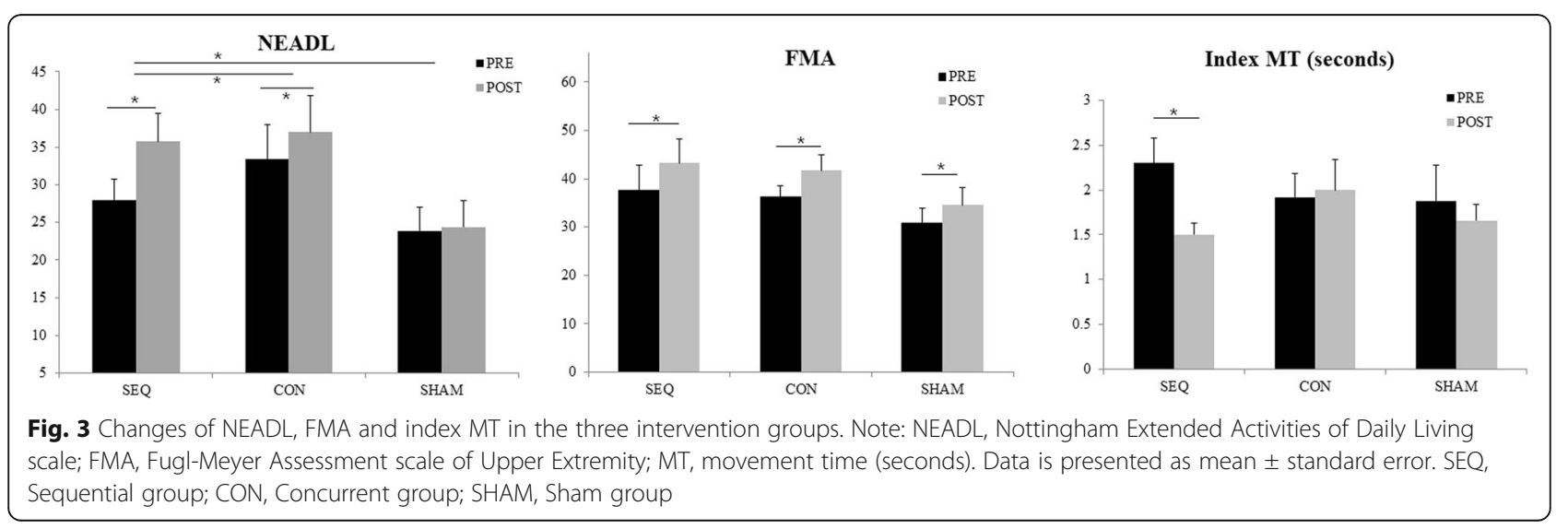


arm because arm performance changes were revealed by kinematic analyses, but not clinical measures. Hence, we recommended future studies to include kinematic assessments to unravel the true add-on effects and timingdependent effects of tDCS with neurorehabilitation interventions regarding paretic arm performance.

Five potential limitations should be considered. First, in view of the clinical characteristics of the enrolled participants, our findings may be applicable only to individuals with mild-to-moderate impairment at the chronic stage. Second, although our study demonstrated timingdependent effects of tDCS with MT on daily function and arm kinematics, it may also be beneficial for future studies to include other outcome measures such as neurophysiological outcomes (e.g., Motor evoked potentials) and neuroimaging outcomes (i.e., brain imaging data). This will help to determine whether the timing of tDCS with MT would affect recovery of neural mechanisms. Third, there were no follow-up assessments. Future studies could evaluate whether the timingdependent effects of tDCS with MT on daily function and hand movement control would be maintained during the follow-up period. Fourth, the functional task training performed in this study was individualized to each participant although the training duration, frequency and categories were standardized for the three groups. Future studies could examine whether the contents of functional task practice would affect treatment effects. Fifth, differential improvements were found in some kinematic outcomes between the three groups although these between-group differences did not reach statistically significant. For example, the normalized total displacement of the index finger and the trunk movement time reduced to the greater extent in the SEQ than the CON and SHAM groups, suggesting potential improvements in the spatial and temporal control of the hand and the trunk primarily in the SEQ group. However, these differences did not reach statistically different between groups, possibly due to the small sample size of this pilot study. Future studies could recruit more participants to determine whether the spatial and temporal kinematic outcomes (i.e., trunk movement time and index movement displacement) would be affected by the timing of tDCS with MT in chronic stroke patients.

\section{Conclusion}

Our study demonstrated potential timing-dependent effects of tDCS with MT on daily function and motor control of paretic hand in chronic stroke patients. Sequentially applying tDCS prior to MT could enhance ADL/IADL function more than applying tDCS concurrently with MT or sham stimulation. Hand movement efficiency also improved in the sequentially combined group. These findings indicated that sequentially combining tDCS prior to MT might be a potentially useful strategy especially for restoration of daily function and paretic hand control for chronic stroke patients, thus may be considered in future clinical application. Further studies with a larger sample size are warranted.

\section{Abbreviations}

NIBS: Non-invasive brain stimulation; tDCS: Transcranial direct current stimulation; MT: Mirror Therapy; ADL: Activities of daily living;

IADL: Instrumental activities of daily living; SEQ: Sequentially combined tDCS with MT; CON: Concurrently combined tDCS with MT; SHAM: Sham tDCS with MT; iM1: Ipsilesional primary motor cortex; EEG: Electroencephalogram; NEADL: Nottingham Extended Activities of Daily Living; FMA: Fugl-Meyer assessment scale of upper extremity; C7: The 7th cervical vertebra; T4: The 4th thoracic vertebra; RT: Reaction time; MT: Movement time;

NTD: Normalized total displacement

\section{Authors' contributions}

WWL and CWC contributed equally to the manuscript. WWL and CWC wrote the first draft of the manuscript. CWC contributed to data analyses. WWL revised and completed the manuscript. CYW contributed to development of the study protocol, grant application, project management and revision of manuscript. All authors involved in interpretation and revision of this study. All authors read and approved the final manuscript.

\section{Funding}

This study was supported by Chang Gung Memorial Hospital (BMRP553), Healthy Aging Research Center, Chang Gung University from the Featured Areas Research Center Program within the Framework of the Higher Education Sprout Project by the Ministry of Education (MOE) in Taiwan (EMRPD1I0451), National Health Research Institutes (NHRI-EX108-10604PI), and the Ministry of Science and Technology (MOST 105-2314-B-182-037MY3, 108-2314-B-182-040-MY3) in Taiwan.

\section{Availability of data and materials}

The datasets used and/or analyzed during the current study are available from the corresponding author on reasonable request.

\section{Ethics approval and consent to participate}

All participants gave their written informed consent prior to participate in this study. Approval of this study was obtained from the Institutional Review Board of Chang Gung Memorial Hospital, Taiyuan, Taiwan.

\section{Consent for publication}

Not applicable.

\section{Competing interests}

The authors declare that they have no competing interests.

\section{Author details}

${ }^{1}$ Department of Occupational Therapy and Graduate Institute of Behavioral Sciences, College of Medicine, Chang Gung University, 259 Wen-hwa 1st Road, Taoyuan City, Taiwan. ${ }^{2}$ Department of Occupational Therapy, I-Shou University, Kaohsiung, Taiwan. ${ }^{3}$ School of Occupational Therapy, College of Medicine, National Taiwan University, Taipei, Taiwan. ${ }^{4}$ Division of Occupational Therapy, Department of Physical Medicine and Rehabilitation, National Taiwan University Hospital, Taipei, Taiwan. ${ }^{5}$ Healthy Aging Research Center, Chang Gung University, Taoyuan, Taiwan. ${ }^{6}$ Department of Physical Medicine and Rehabilitation, Chang Gung Memorial Hospital, Linkou, Taiwan. ${ }^{7}$ Department of Rehabilitation, Taipei Tzu Chi Hospital, The Buddhist Tzu-Chi Medical Foundation, Taipei, Taiwan. ${ }^{8}$ Kaohsiung Chang Gung Memorial Hospital, Kaohsiung, Taiwan. ${ }^{9}$ Graduate Institute of Early Intervention, College of Medicine, Chang Gung University, Taoyuan, Taiwan.

Received: 20 November 2019 Accepted: 6 July 2020

Published online: 20 July 2020

\section{References}

1. Benjamin Emelia J, Muntner P, Alonso A, Bittencourt Marcio S, Callaway Clifton W, Carson April P, et al. Heart disease and stroke statistics-2019 
update: a report from the American Heart Association. Circulation. 2019;139: e56-e528.

2. Billinger SA, Arena R, Bernhardt J, Eng JJ, Franklin BA, Johnson CM, et al. Physical activity and exercise recommendations for stroke survivors: a statement for healthcare professionals from the American Heart Association/American Stroke Association. Stroke. 2014;45:2532-53.

3. Carod-Artal FJ, Egido JA. Quality of life after stroke: the importance of a good recovery. Cerebrovasc Dis. 2009;27:204-14.

4. Claflin ES, Krishnan C, Khot SP. Emerging treatments for motor rehabilitation after stroke. Neurohospitalist. 2015;5:77-88.

5. Pollock A, Farmer SE, Brady MC, Langhorne P, Mead GE, Mehrholz J, et al. Interventions for improving upper limb function after stroke. Cochrane Database Syst Rev. 2014;2014:CD010820.

6. Rensink M, Schuurmans M, Lindeman E, Hafsteinsdóttir T. Task-oriented training in rehabilitation after stroke: systematic review. J Adv Nurs. 2009;65: 737-54

7. Kang N, Summers JJ, Cauraugh JH. Transcranial direct current stimulation facilitates motor learning post-stroke: a systematic review and meta-analysis. J Neurol Neurosurg Psychiatry. 2016;87:345-55.

8. Page SJ, Cunningham DA, Plow E, Blazak B. It takes two: noninvasive brain stimulation combined with neurorehabilitation. Arch Phys Med Rehabil. 2015;96:89-93.

9. Bolognini N, Pascual-Leone A, Fregni F. Using non-invasive brain stimulation to augment motor training-induced plasticity. J Neuroeng Rehabil. 2009;6:8.

10. Thieme H, Morkisch N, Mehrholz J, Pohl M, Behrens J, Borgetto B, et al. Mirror therapy for improving motor function after stroke. Cochrane Database Syst Rev. 2018;7:CD008449.

11. Deconinck FJ, Smorenburg AR, Benham A, Ledebt A, Feltham MG, Savelsbergh GJ. Reflections on mirror therapy: a systematic review of the effect of mirror visual feedback on the brain. Neurorehabil Neural Repair. 2015;29:349-61.

12. Altschuler EL, Wisdom SB, Stone L, Foster C, Galasko D, Llewellyn DME, et al. Rehabilitation of hemiparesis after stroke with a mirror. Lancet. 1999;353: 2035-6.

13. Wu CY, Huang PC, Chen YT, Lin KC, Yang HW. Effects of mirror therapy on motor and sensory recovery in chronic stroke: a randomized controlled trial. Arch Phys Med Rehabil. 2013;94:1023-30.

14. Nitsche MA, Paulus W. Excitability changes induced in the human motor cortex by weak transcranial direct current stimulation. J Physiol. 2000;527:633-9.

15. Stagg CJ, Nitsche MA. Physiological basis of transcranial direct current stimulation. Neuroscientist. 2011;17:37-53.

16. Fleming MK, Rothwell JC, Sztriha L, Teo JT, Newham DJ. The effect of transcranial direct current stimulation on motor sequence learning and upper limb function after stroke. Clin Neurophysiol. 2017;128:1389-98.

17. Hamoudi M, Schambra HM, Fritsch B, Schoechlin-Marx A, Weiller C, Cohen $L G$, et al. Transcranial direct current stimulation enhances motor skill learning but not generalization in chronic stroke. Neurorehabil Neural Repair. 2018;32:295-308.

18. Lefebvre S, Dricot L, Laloux P, Desfontaines P, Evrard F, Peeters A, et al. Increased functional connectivity one week after motor learning and tDCS in stroke patients. Neuroscience. 2017;340:424-35.

19. Rocha S, Silva E, Foerster Á, Wiesiolek C, Chagas AP, Machado G, et al. The impact of transcranial direct current stimulation (tDCS) combined with modified constraint-induced movement therapy (mCIMT) on upper limb function in chronic stroke: a double-blind randomized controlled trial. Disabil Rehabil. 2016;38:653-60

20. Lee SJ, Chun MH. Combination Transcranial direct current stimulation and virtual reality therapy for upper extremity training in patients with subacute stroke. Arch Phys Med Rehabil. 2014;95:431-8.

21. O'Brien AT, Bertolucci F, Torrealba-Acosta G, Huerta R, Fregni F, Thibaut A. Non-invasive brain stimulation for fine motor improvement after stroke: a meta-analysis. Eur J Neurol. 2018;25:1017-26.

22. Stagg C, Jayaram G, Pastor D, Kincses Z, Matthews $P$, Johansen-Berg H. Polarity and timing-dependent effects of transcranial direct current stimulation in explicit motor learning. Neuropsychologia. 2011;49:800-4.

23. Jin M, Zhang Z, Bai Z, Fong KNK. Timing-dependent interaction effects of tDCS with mirror therapy on upper extremity motor recovery in patients with chronic stroke: a randomized controlled pilot study. J Neurol Sci. 2019; 405:116436

24. Cho H-S, Cha H-G. Effect of mirror therapy with tDCS on functional recovery of the upper extremity of stroke patients. J Phys Ther Sci. 2015;27:1045-7.
25. Schwarz A, Kanzler Christoph M, Lambercy O, Luft Andreas R, Veerbeek Janne M. Systematic review on kinematic assessments of upper limb movements after stroke. Stroke. 2019;50:718-27.

26. Lum PS, Mulroy S, Amdur RL, Requejo P, Prilutsky BI, Dromerick AW. Gains in upper extremity function after stroke via recovery or compensation: potential differential effects on amount of real-world limb use. Top Stroke Rehabil. 2009;16:237-53.

27. van Dokkum L, Hauret I, Mottet D, Froger J, Metrot J, Laffont I. The contribution of kinematics in the assessment of upper limb motor recovery early after stroke. Neurorehabil Neural Repair. 2014;28:4-12.

28. Woodbury ML, Velozo CA, Richards LG, Duncan PW. Rasch analysis staging methodology to classify upper extremity movement impairment after stroke. Arch Phys Med Rehabil. 2013;94:1527-33.

29. Rossi S, Hallett M, Rossini PM, Pascual-Leone A. Safety, ethical considerations, and application guidelines for the use of transcrania magnetic stimulation in clinical practice and research. Clin Neurophysiol. 2009:120:2008-39.

30. Hsieh YW, Liing RJ, Lin KC, Wu CY, Liou TH, Lin JC, et al. Sequencing bilateral robot-assisted arm therapy and constraint-induced therapy improves reach to press and trunk kinematics in patients with stroke. J Neuroeng Rehabil. 2016;13:31.

31. Butler AJ, Shuster M, O'Hara E, Hurley K, Middlebrooks D, Guilkey K. A metaanalysis of the efficacy of anodal transcranial direct current stimulation for upper limb motor recovery in stroke survivors. J Hand Ther. 2013;26:162-71.

32. Bastani $A$, Jaberzadeh $\mathrm{S}$. Does anodal transcranial direct current stimulation enhance excitability of the motor cortex and motor function in healthy individuals and subjects with stroke: a systematic review and meta-analysis. Clin Neurophysiol. 2012;123:644-57.

33. Pavlova E, Kuo M-F, Nitsche MA, Borg J. Transcranial direct current stimulation of the premotor cortex: effects on hand dexterity. Brain Res. 2014;1576:52-62.

34. Tedesco Triccas L, Burridge JH, Hughes AM, Pickering RM, Desikan M, Rothwell JC, et al. Multiple sessions of transcranial direct current stimulation and upper extremity rehabilitation in stroke: a review and meta-analysis. Clin Neurophysiol. 2016;127:946-55

35. Russo C, Souza Carneiro MI, Bolognini N, Fregni F. Safety review of Transcranial direct current stimulation in stroke. Neuromodulation. 2017;20: 215-22.

36. Wiethoff $S$, Hamada M, Rothwell JC. Variability in response to transcranial direct current stimulation of the motor cortex. Brain Stimul. 2014;7:468-75.

37. Dyke K, Kim S, Jackson GM, Jackson SR. Intra-subject consistency and reliability of response following $2 \mathrm{~mA}$ Transcranial direct current stimulation. Brain Stimul. 2016;9:819-25.

38. Chew T, Ho KA, Loo CK. Inter- and intra-individual variability in response to Transcranial direct current stimulation (tDCS) at varying current intensities. Brain Stimul. 2015;8:1130-7.

39. Wu CY, Chuang LI, Lin KC, Hong WH. Responsiveness, minimal detectable change, and minimal clinically important difference of the Nottingham extended activities of daily living scale in patients with improved performance after stroke rehabilitation. Arch Phys Med Rehabil. 2011;92:1281-7.

40. Sarker SJ, Rudd AG, Douiri A, Wolfe CD. Comparison of 2 extended activities of daily living scales with the Barthel index and predictors of their outcomes: cohort study within the South London stroke register (SLSR). Stroke. 2012;43:1362-9.

41. Fugl-Meyer AR, Jääskö L, Leyman I, Olsson S, Steglind S. The post-stroke hemiplegic patient. 1. A method for evaluation of physical performance. Scand J Rehabil Med. 1975;7:13-31.

42. Sullivan KJ, Tilson JK, Cen SY, Rose DK, Hershberg J, Correa A, et al. Fugl Meyer assessment of sensorimotor function after stroke: standardized training procedure for clinical practice and clinical trials. Stroke. 2011;42: 427-32.

43. Michaelsen Stella M, Dannenbaum R, Levin MF. Task-specific training with trunk restraint on arm recovery in stroke. Stroke. 2006;37:186-92.

44. Brooks VB, Watts SL. Adaptive programing of arm movements. J Mot Behav. 1988;20:117-32.

45. Wu CY, Lin KC, Chen $\mathrm{HC}$, Chen $\mathrm{IH}$, Hong WH. Effects of modified constraintinduced movement therapy on movement kinematics and daily function in patients with stroke: a kinematic study of motor control mechanisms. Neurorehabil Neural Repair. 2007;21:460-6.

46. Cohen J. Statistical power analysis for the social sciences; 1988 
47. Lee MT, Jang Y, Chang WY. How do impairments in cognitive functions affect activities of daily living functions in older adults? PLoS One. 2019;14: e0218112.

48. Carter LT, Oliveira DO, Duponte J, Lynch SV. The relationship of cognitive skills performance to activities of daily living in stroke patients. Am J Occup Ther. 1988:42:449-55.

49. Mlinac ME, Feng MC. Assessment of activities of daily living, self-care, and Independence. Arch Clin Neuropsychol. 2016;31:506-16.

50. Stoykov ME, Madhavan S. Motor priming in neurorehabilitation. J Neurol Phys Ther. 2015;39:33-42.

51. Schabrun SM, Chipchase LS. Priming the brain to learn: the future of therapy? Man Ther. 2012;17:184-6.

52. Christova M, Rafolt D, Gallasch E. Cumulative effects of anodal and priming cathodal tDCS on pegboard test performance and motor cortical excitability. Behav Brain Res. 2015:287:27-33.

53. Horvath J, Carter O, Forte J. Transcranial direct current stimulation: five important issues we aren't discussing (but probably should be). Front Syst Neurosci. 2014;8:2.

54. Huang YZ, Lu MK, Antal A, Classen J, Nitsche M, Ziemann U, et al. Plasticity induced by non-invasive transcranial brain stimulation: a position paper. Clin Neurophysiol. 2017;128:2318-29.

55. Bortoletto M, Pellicciari MC, Rodella C, Miniussi C. The interaction with taskinduced activity is more important than polarization: a tDCS study. Brain Stimul. 2015:8:269-76.

56. Antal A, Terney D, Poreisz C, Paulus W. Towards unravelling task-related modulations of neuroplastic changes induced in the human motor cortex. Eur J Neurosci. 2007;26:2687-91.

57. Quartarone A, Rizzo V, Bagnato S, Morgante F, Sant'Angelo A, Romano M, et al. Homeostatic-like plasticity of the primary motor hand area is impaired in focal hand dystonia. Brain. 2005;128:1943-50.

58. Thirugnanasambandam N, Sparing R, Dafotakis M, Meister IG, Paulus W, Nitsche MA, et al. Isometric contraction interferes with transcranial direct current stimulation (tDCS) induced plasticity: evidence of state-dependent neuromodulation in human motor cortex. Restor Neurol Neurosci. 2011;29: 311-20.

59. Ang KK, Chua KSG, Phua KS, Wang C, Chin ZY, Kuah CWK, et al. A randomized controlled trial of EEG-based motor imagery brain-computer interface robotic rehabilitation for stroke. Clin EEG Neurosci. 2015;46:310-20.

60. Hesse S, Waldner A, Mehrholz J, Tomelleri C, Pohl M, Werner C. Combined transcranial direct current stimulation and robot-assisted arm training in subacute stroke patients: an exploratory, randomized multicenter trial. Neurorehabil Neural Repair. 2011;25:838-46.

61. Edwards DJ, Cortes M, Rykman-Peltz A, Chang J, Elder J, Thickbroom G, et al. Clinical improvement with intensive robot-assisted arm training in chronic stroke is unchanged by supplementary tDCS. Restor Neurol Neurosci. 2019; 37:167-80.

62. Elsner B, Kwakkel G, Kugler J, Mehrholz J. Transcranial direct current stimulation (tDCS) for improving capacity in activities and arm function after stroke: a network meta-analysis of randomised controlled trials. J Neuroeng Rehabil. 2017;14:95.

63. Giacobbe V, Krebs HI, Volpe BT, Pascual-Leone A, Rykman A, Zeiarati G, et al. Transcranial direct current stimulation (tDCS) and robotic practice in chronic stroke: the dimension of timing. NeuroRehabilitation. 2013;33:49-56.

\section{Publisher's Note}

Springer Nature remains neutral with regard to jurisdictional claims in published maps and institutional affiliations.

Ready to submit your research? Choose BMC and benefit from:

- fast, convenient online submission

- thorough peer review by experienced researchers in your field

- rapid publication on acceptance

- support for research data, including large and complex data types

- gold Open Access which fosters wider collaboration and increased citations

- maximum visibility for your research: over $100 \mathrm{M}$ website views per year

At BMC, research is always in progress.

Learn more biomedcentral.com/submissions 